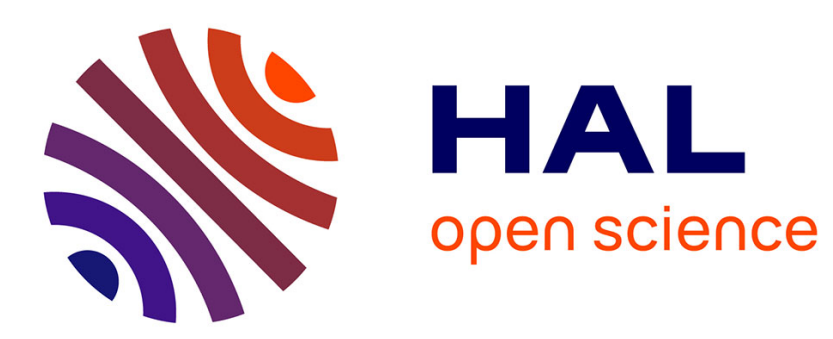

\title{
Prédication et emphase en anglais : le rôle de la prosodie
} Sophie Herment-Dujardin

\section{- To cite this version:}

Sophie Herment-Dujardin. Prédication et emphase en anglais : le rôle de la prosodie. Faits de langues, 2008, 31-32, pp.243-252. hal-00369931

\section{HAL Id: hal-00369931 \\ https://hal.science/hal-00369931}

Submitted on 23 Mar 2009

HAL is a multi-disciplinary open access archive for the deposit and dissemination of scientific research documents, whether they are published or not. The documents may come from teaching and research institutions in France or abroad, or from public or private research centers.
L'archive ouverte pluridisciplinaire HAL, est destinée au dépôt et à la diffusion de documents scientifiques de niveau recherche, publiés ou non, émanant des établissements d'enseignement et de recherche français ou étrangers, des laboratoires publics ou privés. 


\title{
Prédication et emphase en anglais :
}

\section{le rôle de la prosodie}

\author{
Sophie Dujardin-Herment
}

Ce que j'entends par emphase est la mise en relief, d'une façon ou d'une autre d'un ou plusieurs éléments d'un énoncé. Je m'intéresserai à différentes façons de procéder à cette mise en relief, et mettrai en parallèle les niveaux syntaxique et prosodique à l'aide de quelques structures telles que des clivées en wh-, des extrapositions, l'insertion du « do » communément appelé emphatique et également des exemples de focalisation uniquement prosodique. L'analyse aura pour but de voir s'il existe des nuances prédicatives entre ces deux niveaux (syntaxique et prosodique), et lesquelles.

L'emphase strictement prosodique peut-elle être prédicative ? Possède-t-elle des caractéristiques acoustiques récurrentes ? Retrouve-t-on ces caractéristiques lorsqu'une structure syntaxique est présente ? Peut-on parler d'intonation prédicative ?

Telles sont certaines des questions que je poserai et auxquelles je tenterai d'apporter des éléments de réponse.

\section{DEFINITIONS}

Je pars de la notion la plus large de la prédication, comme l'entendent Garnier \& Guimier ${ }^{1}$ qui disent que :

la prédication est l'opération consistant à mettre en relation un sujet et un prédicat.

Selon eux, la prédication peut également désigner

le résultat de cette opération.

\footnotetext{
* Université de Provence, Laboratoire Parole et Langage, CNRS UMR 6057

Courriel : dujardin@up.univ-aix.fr

${ }^{1}$ Garnier, G. \& C. Guimier, 1997, L'épreuve de linguistique au capes et à l'agrégation d'anglais, Nathan Universités.
} 
Ensuite je désigne par emphase la mise en relief, d'une façon ou d'une autre, d'un ou plusieurs éléments d'un énoncé. A nouveau, je prends le terme emphase dans son sens le plus large. L'étude présentée ici s'intéresse à quelques structures de focalisation syntaxique, ainsi qu'à la focalisation purement prosodique. J'inclus donc dans la notion d'emphase la focalisation.

Enfin le terme prosodie désigne les variations de hauteur mélodique, d'intensité, de durée et de rythme.

\section{CORPUS ET METHODE}

Le corpus étudié est de l'anglais uniquement oral, spontané, de la parole naturelle : des enregistrements de radio et des interviews ont été analysés. Ceci est en effet indispensable puisque l'on parle de prosodie. Un des enregistrements parle de mariages arrangés et forcés, c'est un débat entre deux femmes. Un autre est l'interview d'une femme écrivain qui a publié un livre sur le terrorisme. Enfin20deux lecteurs britanniques ont été enregistrés, ils parlent de la France et de leur vie en France.

Je me suis intéressée à l'emphase en lien avec la prédication et ai donc choisi d'étudier quelques structures de focalisation mettant en jeu la prédication.

La question que je me suis posée et à laquelle je vais tenter d'apporter des éléments de réponse est la suivante : les structures de focalisation mettant en jeu la prédication d'une façon ou d'une autre possèdent-elles une prosodie particulière?

\section{FOCALISATION PROSODIQUE}

Ce que $\mathrm{j}$ 'appelle focalisation uniquement prosodique est la mise en relief qui n'utilise aucun procédé syntaxique, mais uniquement des caractéristiques prosodiques.

La mise en relief peut être effectuée grâce à la fréquence fondamentale, c'està-dire aux variations de hauteur mélodique avec des mouvements mélodiques plus amples ou une hauteur plus élevée, comme dans l'exemple (1) dont la courbe de fréquence fondamentale est extraite ci-dessous (figure 1).

(1) Or would you say domestic violence is a private issue ?

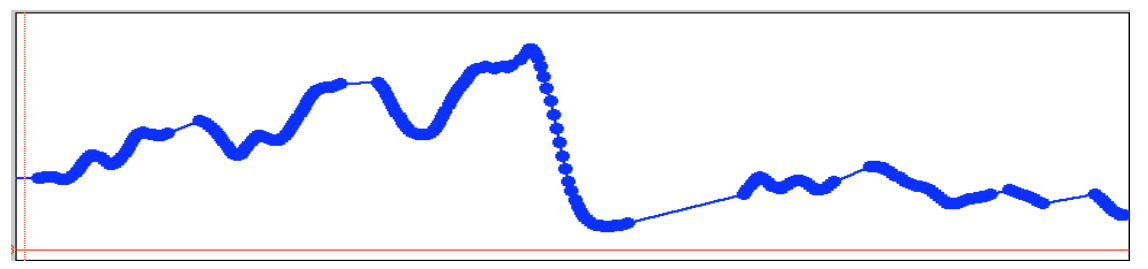

Figure 1 : courbe de fréquence fondamentale pour l'énoncé (1) 
On remarque une montée progressive sur le début de la phrase avec une grande chute qui correspond au mot «violence » puis la fin de la phrase relativement statique grave. Ce schéma intonatif très marqué suffit à mettre en relief le mot « violence » et à rendre l'énoncé emphatique.

On peut aussi jouer sur la durée segmentale, c'est-à-dire la durée des phonèmes, des consonnes et des voyelles. On allonge en général le mot que l'on veut mettre en relief et l'on raccourcit les mots qui l'entourent.

L'intensité peut également varier. Elle augmente souvent avec la hauteur mélodique.

L'attaque du mot emphatique peut être plus marquée : s'il commence par une plosive $(\mathrm{p}, \mathrm{t}, \mathrm{k}, \mathrm{b}, \mathrm{d}, \mathrm{g})$, la tenue de la plosion sera plus longue, ou bien un coup de glotte peut précéder la voyelle initiale, ou encore la consonne initiale peut être allongée par exemple.

Enfin marquer une pause avant ou avant et après un mot peut en augmenter la mise en relief.

J'ai donc cherché à comparer les caractéristiques prosodiques de certaines structures focalisantes liées à la prédication et je les comparerai avec des focalisations uniquement prosodiques pour voir s'il existe des différences.

\section{LES EXTRAPOSITIONS}

Je me suis intéressée aux extrapositions car elles sont une façon de mettre en relief la prédication.

En effet, les extrapositions rencontrées dans le20corpus que j'ai choisi mettent en avant le jugement de l'énonciateur. Celui-ci passe en tête d'énoncé, ce qui lui confère davantage de relief. La deuxième partie de l'énoncé est déjà connue, la prédication est donc déjà posée.

Au niveau prosodique on pourrait s'attendre à une mise en relief d'un élément de la proposition principale par rapport à l'élément déjà connu.

$\mathrm{J}$ 'ai analysé les exemples que j'avais et me suis rendu compte que certaines extrapositions ne forment qu'une seule unité intonative, c'est-à-dire qu'on n'a pas de coupure au niveau intonatif entre les deux parties de l'extraposition :

(2) It's quite hard hitting reports in some ways /

(3) it's very hard hitting them /

(4) It's very clear who we are / and it's very clear who the outsiders are

(5) It was very impressive to see that /

Dans ce cas, l'élément de la principale n'est pas mis en relief prosodiquement alors que l'élément connu l'est. La prédication déjà posée est renforcée. 
Dans d'autres exemples, l'extraposition est découpée en deux unités intonatives :

(6) It's extraordinary / to see to how little attention that case has gotten

(7) that it is an erroneous concept / to think of al-Qaeda as a closed group of people

(8) it seems likely / that the anthrax letter attacks were committed by a single individual

Une rupture mélodique a lieu entre les deux parties de la structure et on a une mise en relief prosodique de l'élément de la principale et de l'élément déjà connu. Les deux prédications sont donc essentielles. L'une est posée, l'autre renforcée.

Aucune des extrapositions étudiées ici ne montre de focalisation prosodique uniquement sur la prédication nouvelle. La prédication déjà posée est toujours réaccentuée, et la prédication nouvelle peut ne pas l'être du tout, ce qui infirme l'hypothèse de départ.

Cela signifie que la structure syntaxique qui renvoie la prédication nouvelle en tête d'énoncé suffit peut-être alors à la mise en relief et une mise en relief prosodique n'est donc pas nécessaire.

Il reste tout de même étonnant que la prédication déjà posée soit toujours réaccentuée. On aurait pu s'attendre au contraire à une désaccentuation de cette deuxième partie de structure extraposée.

\section{LES CLIVEES EN WH-}

Les clivées en wh- méritent d'être analysées également.

Elles annoncent en effet quelque chose d'important à venir. Ce quelque chose est soit inattendu, il s'agit alors d'une nouvelle prédication, soit c'est un fait connu que l'on rappelle, la prédication est déjà posée.

J'ai donc essayé de voir s'il existait des différences prosodiques entre ces deux niveaux

En ce qui concerne les clivées marquant une nouvelle prédication, la prosodie est relativement neutre dans certains cas et plus marquée sans être vraiment très emphatique dans d'autres cas.

Il s'est révélé très difficile de dégager des constantes ici.

Pour les clivées dans lesquelles la prédication est déjà posée, on arrive aux mêmes conclusions :

- on peut avoir une prosodie très marquée, emphatique même, comme en (9) où l'on trouve un mouvement mélodique très ample sur « do » ( $c f$. figure 2 plus bas) :

(9) What I do know is, it's a hidden problem...

On a un élément contrastif après what (I do know) alors que l'on attend normalement du déjà connu. Il y a un verbe20à la forme affirmative ici avec insertion 
de « do », qui marque un contraste dans ce cas précis. C'est après la virgule que l'on a du déjà connu. Je reviendrai sur cet exemple plus loin ( $c f .5$.).

- ou bien la prosodie peut être relativement neutre, comme en (10) ci-dessous où tout est déjà connu aussi et où la mélodie est assez plate :

(10) What seems most apparent to us is that there isn't a simple military solution

A nouveau il s'avère très difficile de dégager des constantes.

On peut tout de même dire qu'il ressort de l'analyse prosodique des clivées en wh- du corpus étudié ici qu'elles possèdent toutes 2 unités intonatives.

Les éléments placés après «what » sont normalement déjà connus, et sont pourtant toujours accentués, puisqu'ils forment dans tous nos exemples une unité intonative, avec donc forcément un accent nucléaire. Mais la prosodie peut être neutre.

La deuxième partie de la clivée forme aussi une unité intonative, et est de la même façon soit neutre, soit appuyée :

(11) so I think what's most important is that we try to undermine the false idea that the Al-Qaeda movement is promoting that the US is out to humiliate the Islamic world

(12) that what globalization means is the humiliation of the Islamic world

En (11), la prédication est nouvelle et pourtant la prosodie est très neutre. La phrase (12) arrive plus tard dans l'interview, la prédication est déjà posée, et pourtant la prosodie est nettement plus marquée avec les mots « means » et surtout « humiliation » très appuyés.

Il semble donc que la syntaxe ait ici le rôle déterminant en ce qui concerne la mise en relief. La prosodie ne vient renforcer cette mise en relief qu'en fonction du message que le locuteur veut faire passer : il peut tout aussi bien augmenter le relief sur la prédication nouvelle grâce à une prosodie emphatique en plus de la structure syntaxique, ou alors choisir d'insister prosodiquement encore sur des faits déjà connus et renforcer du fait la prédication déjà posée (comme c'est le cas en (12)).

Mais dans le cas des clivées, la prosodie n'est pas forcément emphatique. La structure syntaxique, qui est d'ailleurs moins courante en anglais qu'en français, donc remarquable, semble suffire à donner déjà du relief à un élément.

\section{LE « DO » EMPHATIQUE}

Le « do » communément appelé emphatique, aussi nommé d'insistance, réfère à l'auxiliaire qui apparaît dans une forme affirmative.

Au niveau prédicatif, ce « do » marque que le lien entre sujet et prédicat est problématique :

- 《do » vient soit remettre en cause la relation prédicative : il est alors en général contrastif, il nie ce qui a été dit avant,

- $\quad$ soit il vient renforcer au contraire le lien prédicatif. 
Je me suis donc demandé si le traitement accentuel était différent selon les cas.

\section{1. «Do » remet en cause la prédication}

Lorsque « do » remet en cause la prédication, la prosodie est très marquée :

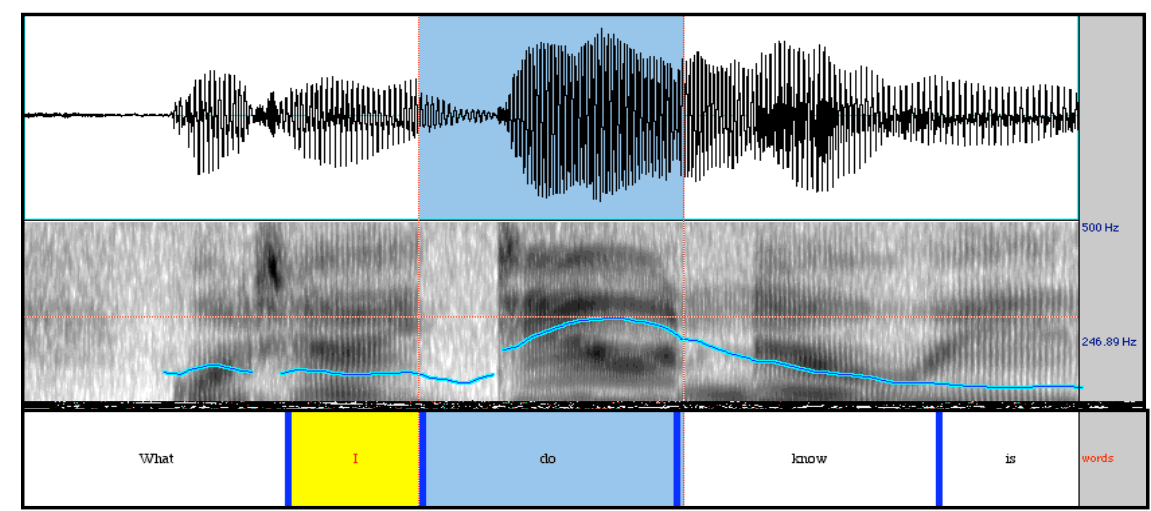

Figure 2 : fenêtre extraite du logiciel PRAAT pour l'énoncé (9)

La figure 2 ci-dessus représente la phrase (9)

(9) What I do know is....

Le haut de la fenêtre est l'oscillogramme qui permet de voir l'amplitude du son. Le spectre est représenté par les tons de gris sous l'oscillogramme. Les sons voisés sont marqués par du gris foncé et le vagues de gris foncé que l'on peut voir sont les formants des voyelles. La courbe qui passe au milieu du spectre est la courbe de fréquence fondamentale, qui permet de visualiser la mélodie de l'énoncé.

La dernière ligne est l'étiquetage en mots. Les barres sont les frontières des mots. L'auxiliaire « do » est sélectionné sur cette fenêtre et l'on voit bien que c'est sur ce mot qu'a lieu le mouvement mélodique le plus important. Il est le pivot de l'énoncé (le noyau) et reçoit donc un accent nucléaire (marqué prosodiquement).

\footnotetext{
${ }^{2}$ Logiciel PRAAT : Boersma, P. \& Weenik, D., 1996, PRAAT : a system for doing ph onetics by computer, Report of the Institute of Phonetic Sciences of the University of Amsterdam, 132. (http://www.praat.org)
} 
L'exemple (13) possède les mêmes caractéristiques (mouvement mélodique ample et accent nucléaire) avec en plus une tenue plus importante de la plosion $\mathrm{du} / \mathrm{d} / \mathrm{de} \ll \operatorname{did} »$ :

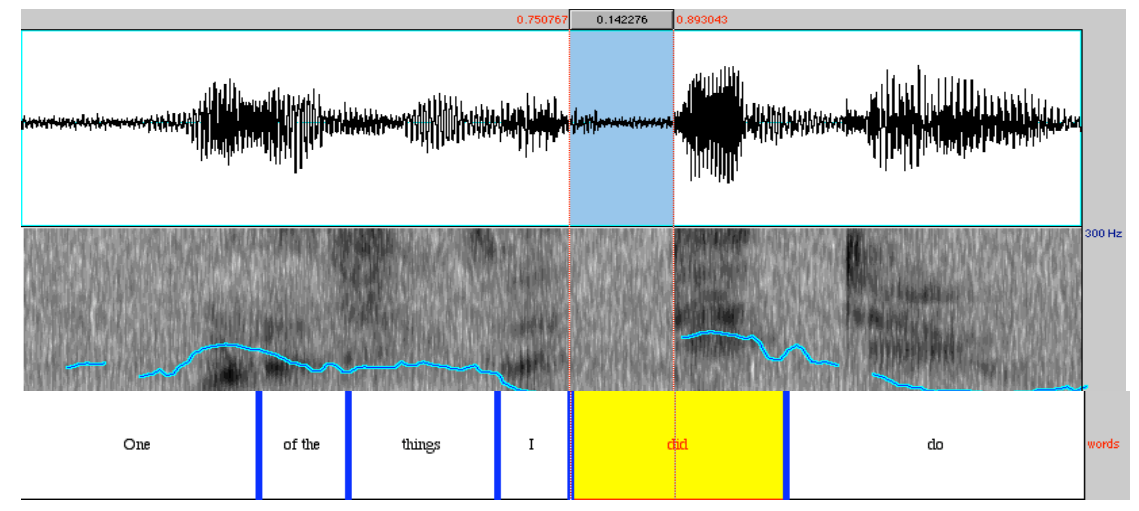

Figure 3 : fenêtre PRAAT pour l'énoncé (13)

(13) one of the things I did do...

La tenue $\mathrm{du} / \mathrm{d} /$ est montrée sur la figure 3 ci-dessus entre les deux lignes verticales. Le logiciel PRAAT permet d'en mesurer la durée qui est ici de 140 millisecondes. Pour donner un élément de comparaison, j'ai mesuré chez ce même locuteur la tenue d'autres consonnes /d/ sur des mots où /d/ se trouvait à l'initiale accentuée : $40 \mathrm{~ms}$ pour «different », $50 \mathrm{~ms}$ pour le mot «definitely » et même seulement $70 \mathrm{~ms}$ sur la négation «don't » (sachant que les négations sont toujours particulièrement accentuées au niveau de la phrase et pas seulement du mot). Il s'agit ici d'un procédé d'emphase prosodique (comme décrit en 3.).

Nous venons donc de voir que lorsque « do » remet en cause la prédication, il est très marqué prosodiquement. Comparons maintenant avec des focalisations uniquement prosodiques portant sur des négations, puisqu'il s'agit en fait du même principe : la prédication est niée par la négation.

Les énoncés (14) et (15), dont les courbes de fréquence fondamentale sont extraites, illustrent ce phénomène : 
(14) Also various movements that are not terrorist groups per se

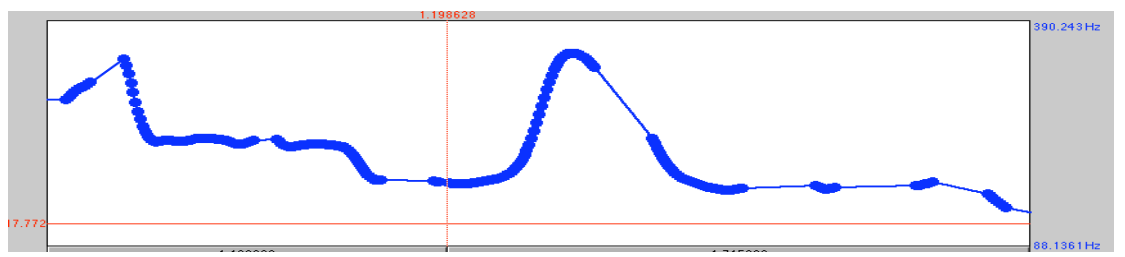

Figure 4 : courbe de FO pour (14)

(15) Why do you thonk they don't get the same kind of press coverage?

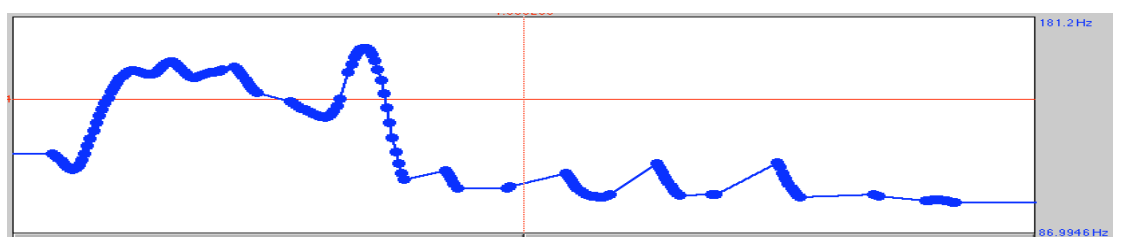

Figure 5 : courbe de F0 pour (15)

On retrouve pour ces deux exemples exactement les mêmes structures prosodiques que pour les énoncés avec un «do » emphatique remettant en cause la prédication : mouvement mélodique très ample et accent nucléaire (sur les négations en (14) et (15)).

Il est intéressant de comparer également avec des emphases purement prosodiques (sans négations) : les énoncés (16) à (18) en sont des exemples et les mots emphatiques sont soulignés.

(16) Le's talk about what you did

(17) And what makes us different from them

(18) That's what you do

On retrouve à nouveau les mêmes caractéristiques, à savoir accent nucléaire et mouvement mélodique ample. La courbe de F0, très révélatrice, a été extraite pour (18) uniquement :

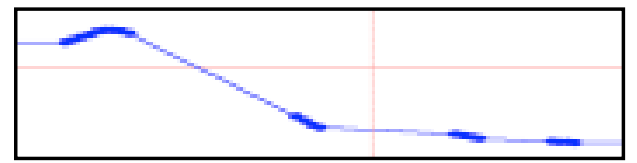

Figure 6 : courbe de F0 pour (18) 
Ainsi les mêmes caractéristiques prosodiques sont présentes pour des contrastes ne portant pas sur la prédication.

\section{2. «Do » renforce la prédication}

Nous avons vu que le « do » emphatique peut soit remettre en cause la prédication, soit renforcer au contraire le lien prédicatif. Plusieurs exemples dans le corpus choisi illustrent le deuxième point et les caractéristiques prosodiques sont alors différentes. Il faudrait bien entendu le contexte mais les exemples (19) à (22) sont tous des cas où la prédication est bien renforcée et non niée :
(19) I do like France very much
(20) I did talk at lenght
(21) But you do interview a domestic terrorist in this book
(22) You do come

Dans tous ces énoncés, la prosodie est marquée, elle n'est pas neutre, mais il est très intéressant de constater que le mouvement mélodique est moins ample que dans les énoncés où «do » remet en cause la prédication, et surtout que « do » ne porte plus l'accent nucléaire, il est tonique de tête de l'énoncé (c'est-àdire premier accent de l'énoncé au niveau suprasegmental, en général statique haut, alors que l'accent nucléaire est le dernier accent de l'énoncé, en général porteur d'un mouvement mélodique ou au début de ce mouvement).

Pour (19) par exemple, le noyau de l'énoncé est «much», en (20) l'accent nucléaire est sur « length», en (21) sur «book» et en (22) sur « come ». La courbe de F0 pour (22) est montrée figure 7 :

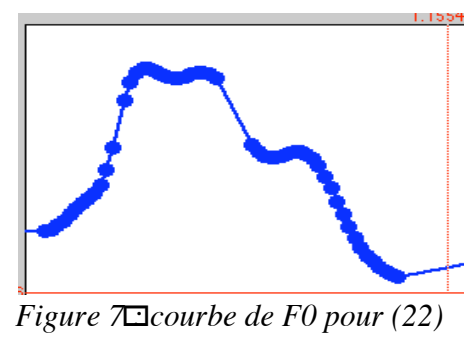

Le plateau dans l'aigu au début de l'énoncé correspond à « do », qui est tonique de tête, et la chute est sur « come » qui est le noyau de l'énoncé.

J'ai alors regardé si l'on retrouvait ces constantes prosodiques dans des énoncés présentant une focalisation uniquement prosodique sur des auxiliaires autres que « do » mais dans le même contexte de renforcement du lien prédicatif : énoncés (23) à (25).

(23) You can actually communicate in their language

(24) Which communities here and on the subcontinent / are keeping well hidden 
(25) And it is theatre

On observe exactement le même phénomène : l'auxiliaire est porteur de la tonique de tête. Le noyau est plus à droite. En (23) le noyau est «language », en $(24)$ « hidden » et en $(25)$ « theatre » comme le montre la figure 8 ci-dessous :

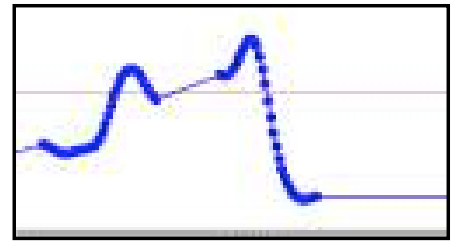

Figure 8 : courbe de F0 pour (25)

Les courbes des figures 7 et 8 se ressemblent: l'auxiliaire est dans l'aigu ( « is » toutefois moins statique que « do ») et le nucleus est final et porteur d'une ample chute.

\subsection{Résumé et conclusion pour le « do » emphatique}

L'analyse prosodique d'énoncés contenant un « do » emphatique s'est montrée très intéressante en lien avec la prédication car elle a permis de dégager deux schémas prosodiques différents :

- lorsque «do » remet en cause la relation prédicative, il est noyau de l'énoncé, fortement marqué prosodiquement. Il possède les mêmes caractéristiques prosodiques que d'autres éléments contrastifs, et en particulier la négation.

- lorsque « do » renforce la prédication, il est moins marqué prosodiquement. Il est tonique de tête de l'unité intonative. Il possède les mêmes caractéristiques prosodiques que d'autres éléments jouant le même rôle sans intervention de la syntaxe.

Mais au-delà de ces phénomènes le fait de comparer des énoncés contenant un « do » emphatique avec des énoncés ne contenant pas d'insertion syntaxique mais des caractéristiques prédicatives équivalentes permet donc d'affirmer que la prosodie reste la même, qu'il y ait insertion ou non d'un élément au niveau syntaxique.

\section{CONCLUSION GENERALE}

L'étude décrite ici n'est qu'une ébauche et mériterait d'être approfondie. Il faudrait travailler sur davantage d'exemples, mais on peut tout de même dire que l'on entrevoit des pistes intéressantes.

La syntaxe et la prosodie sont deux domaines complémentaires, on l'a vu, dont les liens, extrêmement complexes, restent encore largement à explorer. Dans certains cas la syntaxe seule semble suffire à mettre un élément en relief comme 
dans les extrapositions ou certaines clivées en wh-. Dans d'autres cas la prosodie seule permet une mise en relief. Et parfois les deux phénomènes se combinent. Le lien avec la prédication rend cette combinaison encore plus subtile, la prosodie venant souvent renforcer le nœud prédicationnel déjà marqué syntaxiquement, comme dans certaines clivées en wh-. Mais nous avons aussi vu lors de l'analyse du « do » emphatique que la prosodie peut rester la même avec ou sans insertion syntaxique. On pourrait alors se risquer à dire qu'il existerait un certain schéma prosodique lié à la prédication, selon que celle-ci est niée ou renforcée.

Je crois enfin qu'il est impossible de ne pas prendre en compte les stratégies discursives individuelles des locuteurs dans ce type d'étude car on a vu qu'il est tout de même parfois dur de dégager des constantes et c'est probablement précisément à cause de ces stratégies. N'oublions pas que nous traitons d'oral, de parole authentique, de discours spontané, ce qui rend la tâche difficile mais d'autant plus passionnante. 